\title{
Commentary
}

\section{Data Generated by Quantitative Liquid Chromatography-Mass Spectrometry Proteomics Are Only the Start and Not the Endpoint: Optimization of Quantitative Concatemer-Based Measurement of Hepatic Uridine-5'-Diphosphate-Glucuronosyltransferase Enzymes with Reference to Catalytic Activity ${ }^{[]}$}

\author{
Brahim Achour, Alyssa Dantonio, Mark Niosi, Jonathan J. Novak, Zubida M. Al-Majdoub, \\ Theunis C. Goosen, Amin Rostami-Hodjegan, and Jill Barber \\ Centre for Applied Pharmacokinetic Research, Division of Pharmacy and Optometry, University of Manchester, Manchester, United \\ Kingdom (B.A., Z.M.A.-M., A.R.-H., J.B.); Department of Pharmacokinetics, Dynamics, and Metabolism, Pfizer Inc., Groton, \\ Connecticut (A.D., M.N., J.J.N., T.C.G.); and Simcyp Limited (a Certara Company), Blades Enterprise Centre, Sheffield, United \\ Kingdom (A.R.-H.)
}

Received December 13, 2017; accepted March 22, 2018

\section{ABSTRACT}

Quantitative proteomic methods require optimization at several stages, including sample preparation, liquid chromatographytandem mass spectrometry (LC-MS/MS), and data analysis, with the final analysis stage being less widely appreciated by end-users. Previously reported measurement of eight uridine-5'-diphosphoglucuronosyltransferases (UGT) generated by two laboratories [using stable isotope-labeled (SIL) peptides or quantitative concatemer (QconCAT)] reflected significant disparity between proteomic methods. Initial analysis of QconCAT data showed lack of correlation with catalytic activity for several UGTs $(1 \mathrm{A4}, 1 \mathrm{~A} 6,1 \mathrm{~A} 9,2 \mathrm{~B} 15)$ and moderate correlations for UGTs $1 \mathrm{~A} 1,1 \mathrm{~A} 3$, and 2B7 $(R s=0.40-0.79$, $P<0.05 ; R^{2}=0.30$ ); good correlations were demonstrated between cytochrome P450 activities and abundances measured in the same experiments. Consequently, a systematic review of data analysis, starting from unprocessed LC-MS/MS data, was undertaken, with the aim of improving accuracy, defined by correlation against activity. Three main criteria were found to be important: choice of monitored peptides and fragments, correction for isotope-label incorporation, and abundance normalization using fractional protein mass. Upon optimization, abundance-activity correlations improved significantly for six UGTs ( $R$ s $=0.53-0.87, P<0.01 ; R^{2}=0.48-0.73$ ); UGT1A9 showed moderate correlation ( $\left.R \mathrm{~s}=0.47, P=0.02 ; R^{2}=0.34\right)$. No spurious abundance-activity relationships were identified. However, methods remained suboptimal for UGT1A3 and UGT1A9; here hydrophobicity of standard peptides is believed to be limiting. This commentary provides a detailed data analysis strategy and indicates, using examples, the significance of systematic data processing following acquisition. The proposed strategy offers significant improvement on existing guidelines applicable to clinically relevant proteins quantified using QconCAT.

\section{Introduction}

Robust quantification of proteins involved in drug pharmacokinetics is required for reliable in vitro-in vivo extrapolation of drug-related outcomes (Al Feteisi et al., 2015b). Various quantitative proteomic strategies share several key steps: 1) selection of signature peptides that represent target proteins in biologic samples, with stable isotopelabeled (SIL) versions used as standards, 2) isolation of tissue fractions that contain these proteins, 3) sample preparation for mass spectrometry, by solubilization and proteolysis of proteins into peptides,

https://doi.org/10.1124/dmd.117.079475

SThis article has supplemental material available at dmd.aspetjournals.org. and 4) simultaneous analysis of standard and native peptides by liquid chromatography-tandem mass spectrometry (LC-MS/MS) (Wegler et al., 2017). Furthermore, using concatenated standards [quantitative concatemer (QconCAT)] requires additional steps to ensure that standard proteins are successfully expressed and sufficiently labeled, purified, and digested (Russell et al., 2013).

Uridine-5'-diphospho-glucuronosyltransferases (UGTs) have recently attracted more clinical attention (Guillemette et al., 2014), leading to increased interest in UGT abundance and activity data (Margaillan et al., 2015). Our laboratories previously reported comparability of two proteomic assays: in-solution sample preparation with quantification using SIL peptide standards (Fallon et al., 2013) and gel-based sample preparation with QconCAT proteomics (Achour et al., 2014b).

ABBREVIATIONS: BCA, Bicinchoninic acid assay; HLM, human liver microsomes; LC-MS/MS, liquid chromatography-tandem mass spectrometry; MRM, multireaction monitoring; PBPK, physiologically based pharmacokinetics; QconCAT, quantification concatemer; SIL, stable isotope-labeled; UGT, uridine-5' -diphospho-glucuronosyltransferase. 
Disparities between abundances generated by these methods pointed to the necessity of validating measurements using UGT-isoform-specific activity. Reliable correlation was demonstrated for the SIL-based measurements; discrepancies remained for the QconCAT-based dataset (Achour et al., 2017b).

The QconCAT methodology has been validated in various contexts (Scott et al., 2016), including, most notably, cytochrome P450 quantification carried out with the UGT measurements (Achour et al., 2014b). Quantification of UGTs led to complications that had not been observed with bacterial (Al-Majdoub et al., 2014) and yeast samples (Brownridge et al., 2011). The proteomic strategy used in this study is inherently complex; however, many steps have been taken to validate the LC-MS/MS multireaction monitoring (MRM) assay, including assessment of precision and accuracy of measurements, as well as the associated analytical and technical errors (Achour et al., 2014b). Data acquisition constitutes only the first step of data processing, with several subsequent stages aimed at converting these data into abundance levels and then making sense of such levels. These tasks include deciding which elements of the raw data should be used, normalization processes, and quality control checks as applicable. Discrepancies arising at the data analysis stage are not widely appreciated by end-users of proteomic data, especially modelers, and therefore warrant more attention. The aim of this commentary is to highlight the impact of optimizing UGT-specific quantitative factors at the level of data
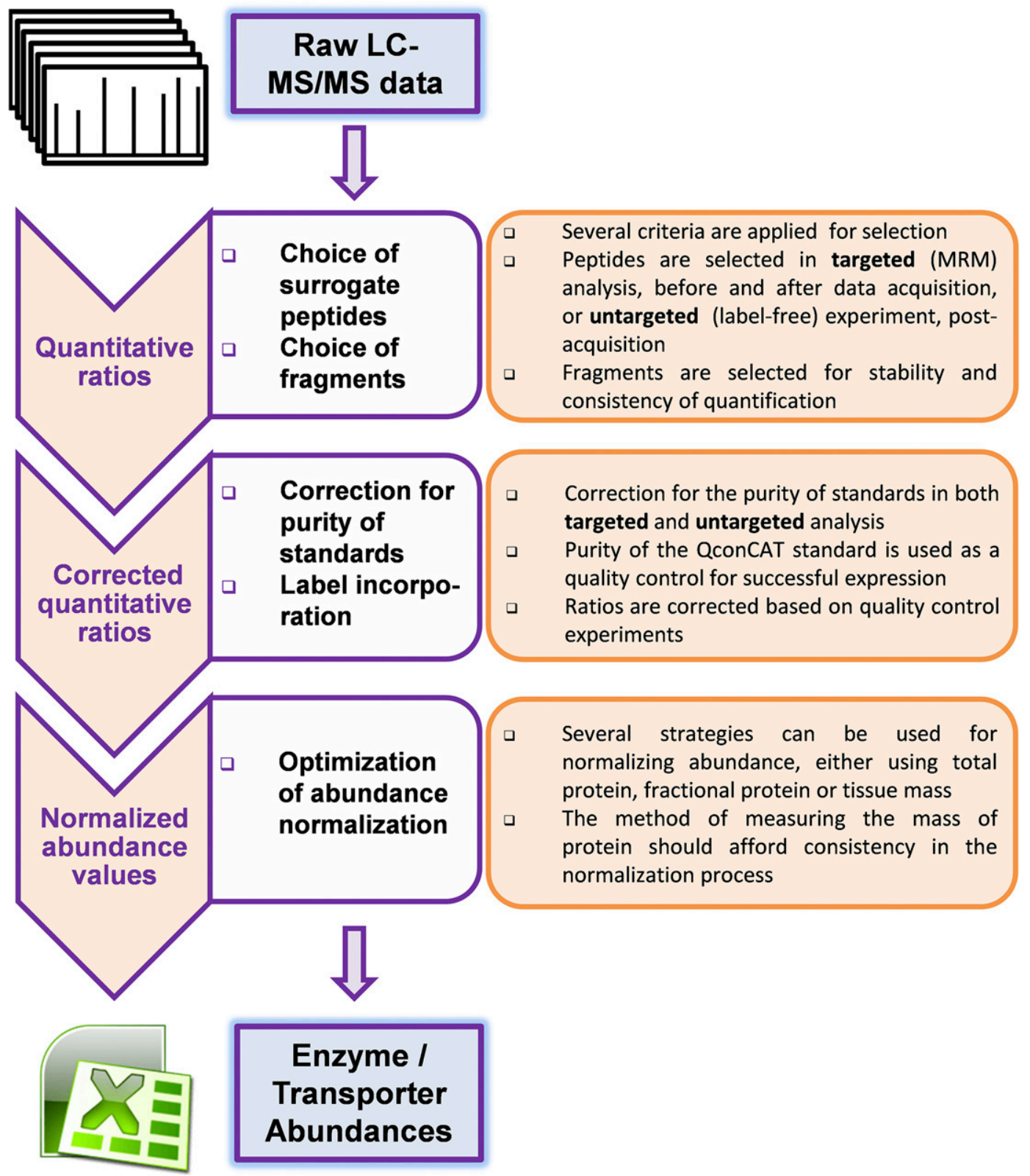

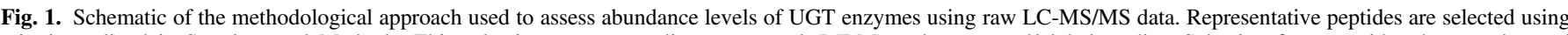

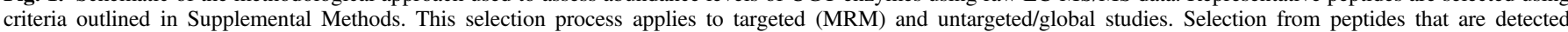

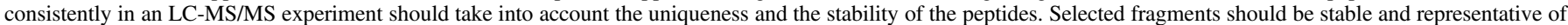

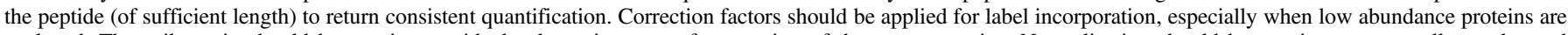

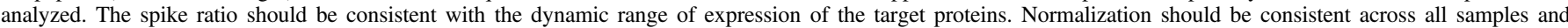
measured parameters. An example of this process is shown in Fig. 2. 
A

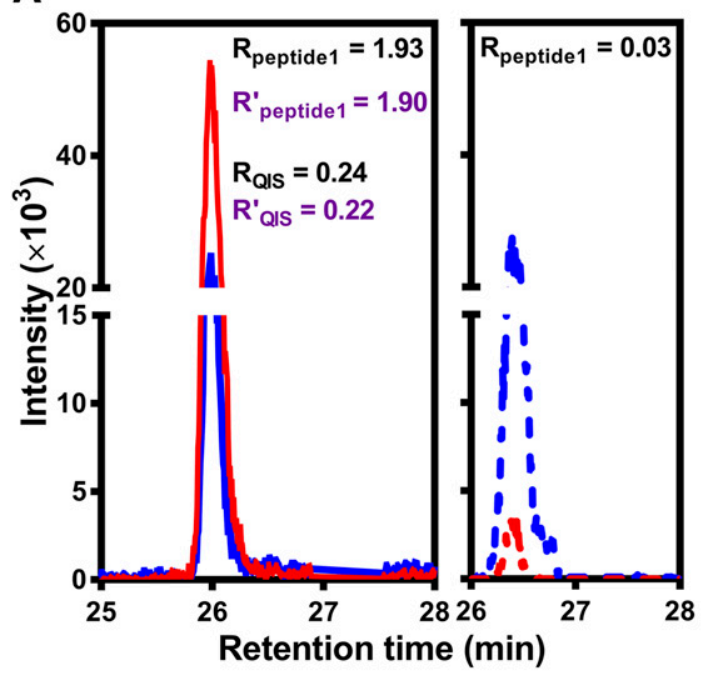

B

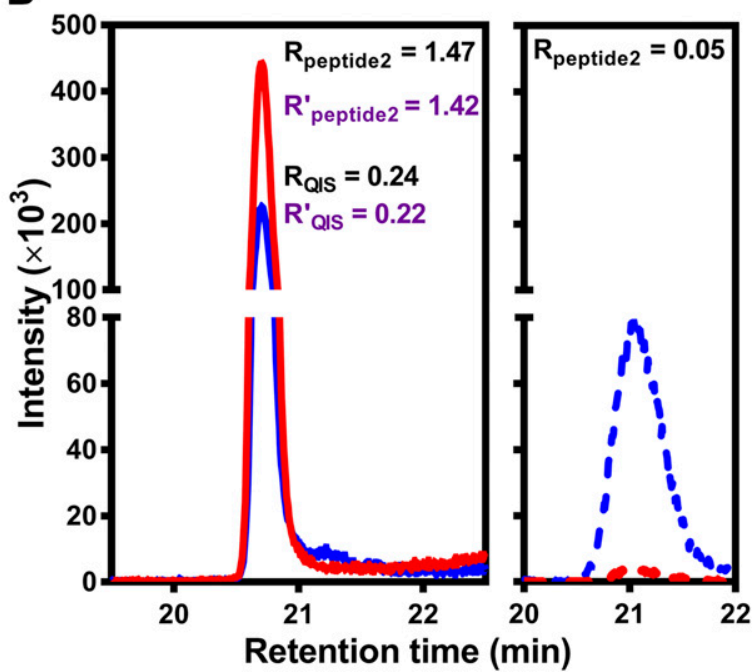

C

D
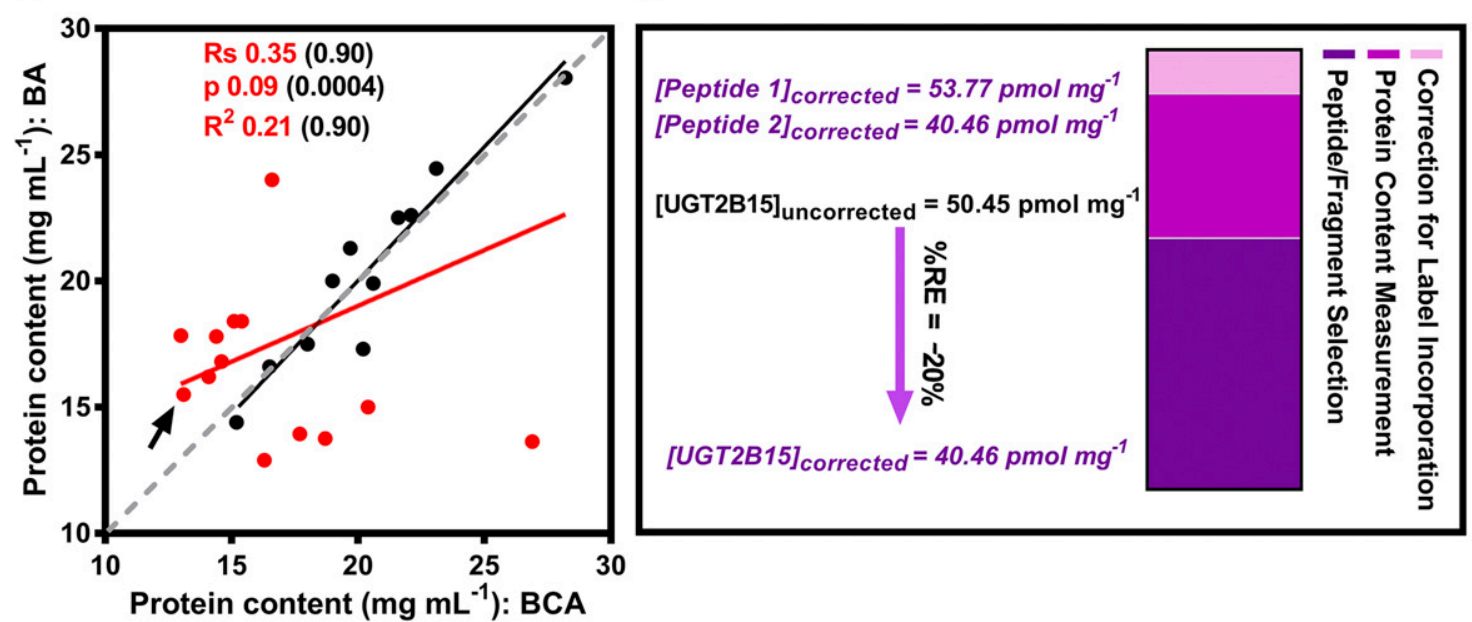

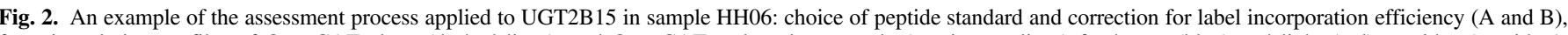

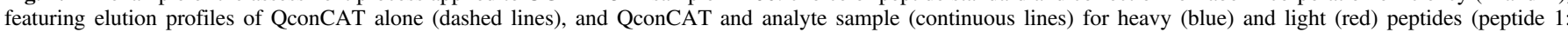

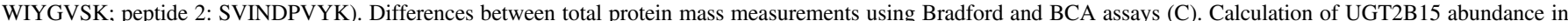

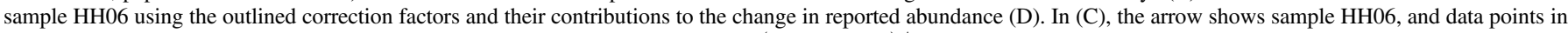

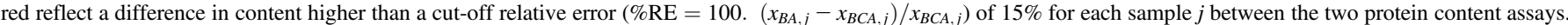

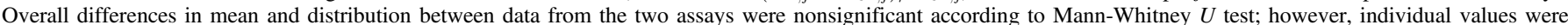

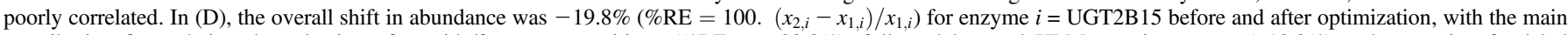

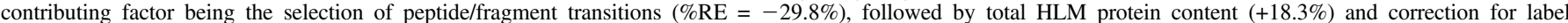
incorporation $(+6.1 \%)$. Text in purple font reflects corrected values. BA, Bradford assay; QIS, QconCAT-based internal standard.

analysis on QconCAT-measured abundances with reference to catalytic activity.

\section{Reassessment of the Methodological Workflow}

Human liver microsomal (HLM) samples $(n=24)$ and methods used to measure UGT abundance and activity were previously described (Achour et al., 2017b). Briefly, eight UGTs (1A1, 1A3, 1A4, 1A6, 1A9, 2B4, 2B7, and 2B15) were independently quantified using QconCAT (Achour et al., 2014b) and SIL-peptide standards (Fallon et al., 2013). Activities of seven enzymes were measured by monitoring the glucuronides of substrates: $\beta$-estradiol (UGT1A1), chenodeoxycholic acid (UGT1A3), trifluoperazine (UGT1A4), 5-hydroxytryptophol (UGT1A6), propofol (UGT1A9), zidovudine (UGT2B7), and $S$-oxazepam (UGT2B15).

Initial QconCAT-based quantification did not show considerable correlation with catalytic activity and therefore required systematic assessment of several data analysis steps. Figure 1 shows a schematic of the data assessment strategy, with a practical example shown in Fig. 2 (for UGT2B15 in sample HH06). Reanalysis of elution profiles and fragment-based quantitative ratios was done using Skyline 3.7 (MacCoss Laboratory Software, Seattle, WA). Measured abundances were reassessed against activity data. An outline of the reassessment strategy is described below.

Choice of Peptide Standards. The peptides that constitute the QconCAT were previously selected on the basis of experimental design followed by theoretical assessment. This approach was limited by options in a data-dependent experiment yielding one to two peptides per UGT (Russell et al., 2013). Extensive sequence homology between UGTs also contributed to this limitation. Initially, peptides that provided higher abundance were used in line with widely accepted literature (Brownridge et al., 2011; Lawless et al., 2016). Instead, we propose 


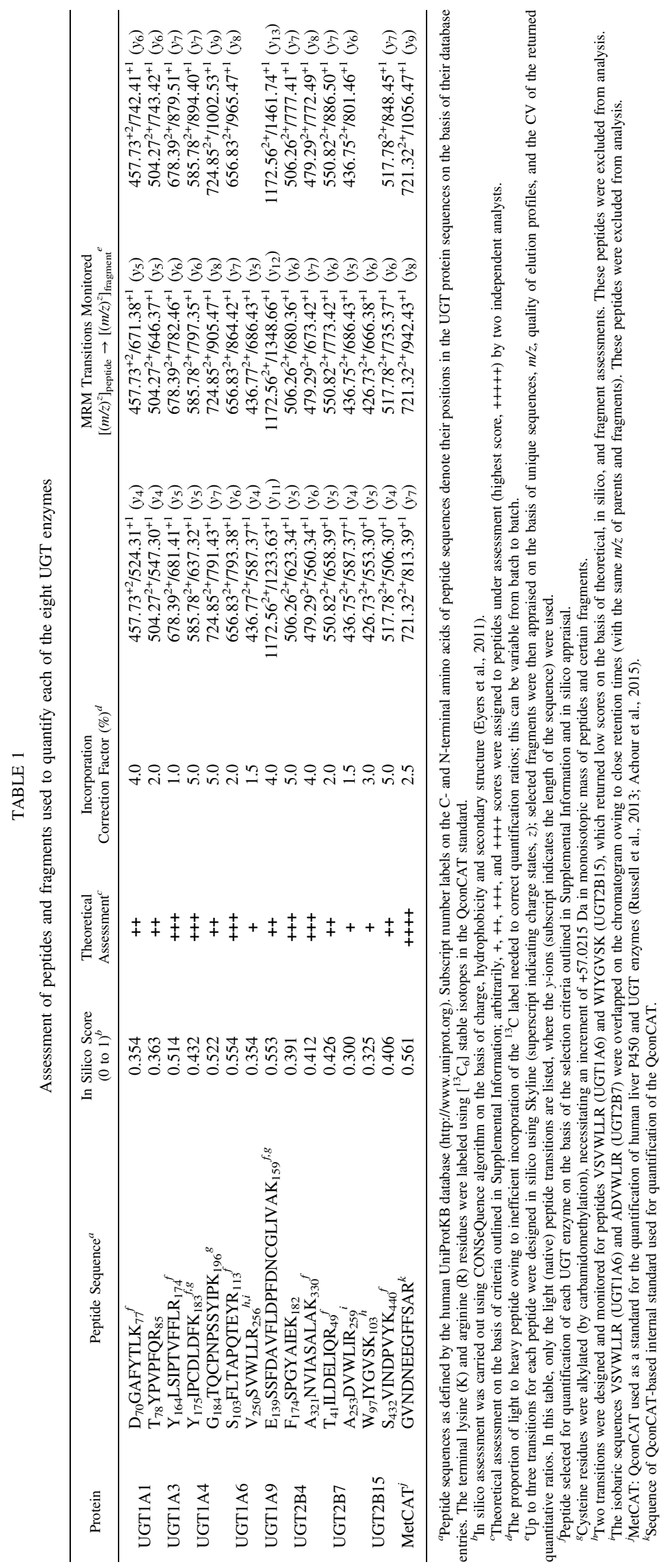


adoption of a better appraisal of quantification that uses as a basis several peptides for each UGT. In this work, assessment of suitability of peptides was carried out on the basis of theoretical appraisal by two independent analysts and in silico evaluation using CONSeQuence algorithm (Eyers et al., 2011). Quantification was subsequently considered on the basis of the more favorable peptide choice (Table 1). Theoretical criteria for peptide assessment are included in Supplemental Methods. Briefly, selected peptides should have unique sequences, and mass-to-charge ratios (of parent and fragment), should not be mapped to membrane-associated domains or subject to polymorphisms or biologic (post-translational) modifications, should be readily cleavable, of suitable length (6-20 amino acids), and have favorable stability (to chemical modification owing to handling/storage) and moderate hydrophobicity (Kamiie et al., 2008; Carroll et al., 2011). Choice of monitored peptide charge state $(z=+2$ or +3$)$ was also considered.

Choice of Peptide Fragments. Fragment selection was initially conducted in silico using Skyline 1.4, with fragment ratios expected to return consistent quantification. Initially, two to three transitions per peptide were monitored with mean ratios being used for quantification. In this work, the uniqueness of fragment sequences and consistency between estimates on the basis of monitored transitions were assessed, especially for low abundance proteins; less-specific fragments returning inconsistent ratios were excluded from analysis. In addition, the chromatographic trace of different fragment ions was assessed and poor quality signals were excluded. Since retention time and $\mathrm{m} / \mathrm{z}$ values monitored had relatively large filters in the LC-MS/MS assay, the $\mathrm{m} / \mathrm{z}$. values for the selected fragments were assessed, with isobaric and isomeric fragments being excluded (Table 1).

Correction for Efficiency of Label Incorporation. Assessment of efficiency of ${ }^{13} \mathrm{C}$-label incorporation into QconCAT protein synthesis was previously reported as an in-house quality control step in QconCAT expression; constructs of $\geq 95 \%$ purity are accepted as quantitative standards (Achour et al., 2015). The level of incorporation can vary batch-to-batch, and impurity is expected to affect quantification, especially of low abundance proteins (Carr et al., 2014). Uncorrected and corrected quantitative ratios were generated using eqs. 1 and 2, respectively (Fig. 2, A and B):

$$
\begin{gathered}
R_{\text {peptide } \frac{L}{H}}=\frac{I_{L_{\text {Peptide }}}}{I_{H_{Q c o n C A T}}} \\
R_{\text {peptide } \frac{L}{H}}^{\prime}=\frac{I_{L_{\text {Peptide }}}-I_{L_{Q c o n C A T}}}{I_{H_{Q c o n C A T}}}
\end{gathered}
$$

where $R_{\text {peptide } \frac{L}{H}}$ and $R_{\text {peptide } \frac{L}{H}}$ are uncorrected and corrected ratios, respectively, used to quantify a peptide representing a UGT enzyme or the QconCAT (using a QconCAT-based internal standard); $I_{L_{\text {Peptide }}}$ is the intensity of the light peptide signal measured in the quantitative experiment; $I_{L_{Q \text { ConCAT }}}$ is the peak intensity of the light peptide originating from the QconCAT measured in quality control experiments; and $I_{H_{\text {QconCAT }}}$ is the signal intensity of the heavy QconCAT peptide measured in the quantitative experiment.

Normalization of Abundance Measurements. Normalization is commonly applied relative to protein mass, leading to abundance levels expressed in units of picomoles per milligram of fractional protein. Protein mass measurement is normally done by a colorimetric assay, generating data that may not be reproducible. Commonly used assays include: Bradford assay (Bradford, 1976), bicinchoninic acid (BCA) assay (Smith et al., 1985), and tryptophan fluorescence assay (Wiśniewski and Gaugaz, 2015). Abundance and activity were measured by independent laboratories and different protein assays were used (Bradford and BCA assays), demonstrating differences in reported contents for matched samples (Fig. 2C). In this study, a proposed approach to resolving this issue was to normalize abundance and activity data using protein levels measured by the same assay (BCA assay), using eq. 3.

$$
[\text { Enzyme }]=[Q I S] \cdot \frac{\mathrm{R}_{\text {Peptide }_{\mathrm{H}}}^{\prime}}{\mathrm{R}_{\mathrm{QIS}_{\mathrm{H}}}^{\prime}} \cdot \frac{F_{v}}{\text { Protein Mass }}
$$

where [Enzyme] is target enzyme abundance (expressed in picomoles per milligram of HLM protein); $[Q I S]$ represents the concentration of the unlabeled internal standard used to quantify the QconCAT [a Glu ${ }^{1}$-fibrinopeptide B analog, modified to reduce the incidence of missed cleavage owing to the glutamate at the N-terminus (Lawless and Hubbard, 2012)]; $F_{v}$ is a volume correction factor relating the analyzed volume to the volume of HLM sample; and Protein Mass is the protein content determined for each sample (BCA assay). The terms assessed in this report were: target peptide and QconCAT-basedinternal-standard ratios (parent-fragment selection and correction for label incorporation) and protein mass used for normalization (Fig. 2D).

Statistical Assessment of the Optimization Process. Correlations were assessed at each stage using Spearman correlation test $(R \mathrm{~s})$ and scatter of data was assessed with linear regression $\left(R^{2}\right)$. The following criteria were used: $\alpha$-value of 0.05 (Bonferroni-corrected for correlation matrices), strong correlation $(R \mathrm{~s}>0.50)$ and limited scatter $\left(R^{2}>0.30\right)$, taking into account the effect of abundance/activity units, as previously advocated (Achour et al., 2017a).

\section{Effects of Systematic Appraisal on Endpoint Measurements}

To generate reliable proteomic data for in vitro-in vivo extrapolationphysiologically based pharmacokinetics, best practice must be ensured throughout the entire quantitative workflow, including data processing. It is important to note that sample preparation and LC-MS/MS methods have previously undergone quality evaluation and returned precise and accurate quantification of cytochromes $\mathrm{P} 450$ in relation to genotype and activity (Achour et al., 2014b). Therefore, only factors specifically affecting UGT measurements were considered.

Effects of the Choice of Monitored Peptides/Fragments. Mass spectrometry-based proteomic strategies rely on using peptides as surrogates for proteins, and the limitations of this approach are still being uncovered. In eukaryotes, protein truncation and splice variants can result in misleading measurements, and technical issues include signal overlap and variable peptide responses between runs. QconCAT design normally follows a pragmatic approach, with two or more peptides included for each protein (Pratt et al., 2006); however, only one peptide is ultimately used for quantification. Two peptides representing each UGT were therefore included in the QconCAT whenever possible. Table 1 shows sequences used in data acquisition, scored on the basis of theoretical and in silico criteria; Supplemental Fig. 1 shows peptide elution profiles. Theoretical assessment of peptide suitability involves consideration of several parameters, which tend to be prioritized somewhat subjectively, and was therefore conducted by two independent analysts; the analysts' scores were in agreement and compared well with in silico assessment. Three pairs of peptides (representing UGTs 1A1, 1A4, and 2B7) returned consistent quantification (Supplemental Fig. 2), whereas three pairs (UGTs 1A6, 2B4, and 2B15) showed significant differences.

When two or more peptides are used to quantify a protein, preference has conventionally been given to peptides that return higher concentrations (Brownridge et al., 2011; Lawless et al., 2016); the assumption is that underestimation can occur owing to differences in efficiency of release of peptides. It has recently become clear that several peptiderelated factors can affect measurements and should therefore be considered when peptide choice is made, ideally in the design stage, although this is not always possible, especially in global proteomics. The 
A

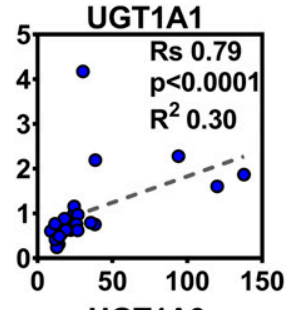

UGT1A3
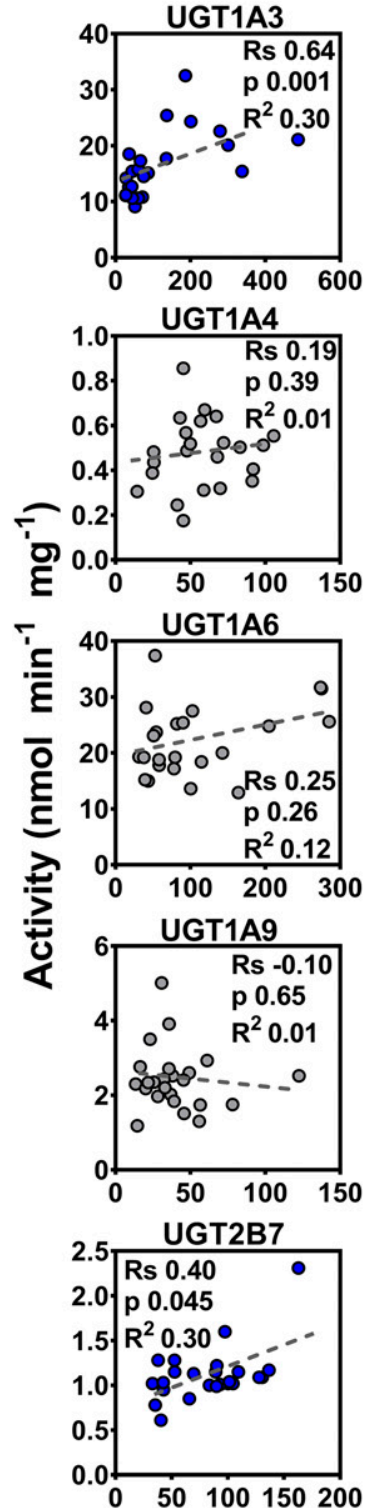

UGT2B15

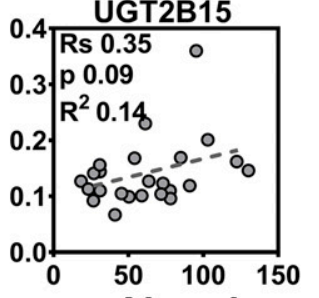

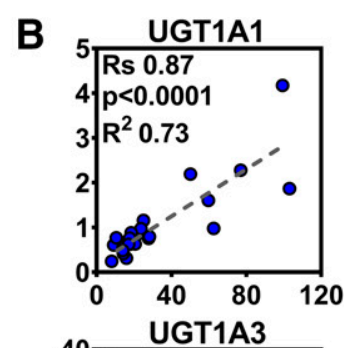
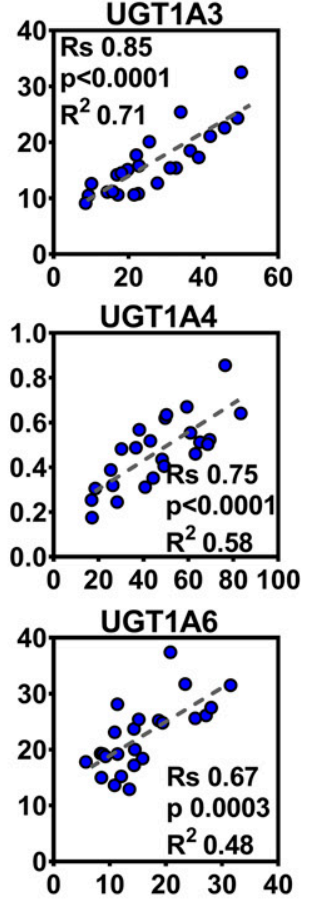

UGT1A9
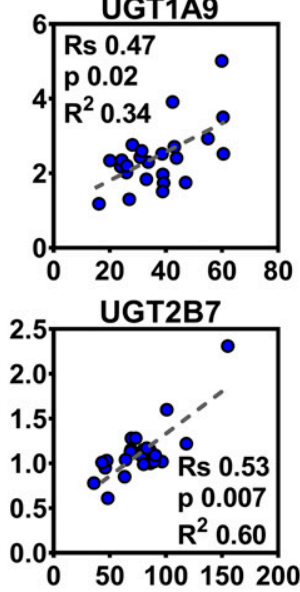

UGT2B15

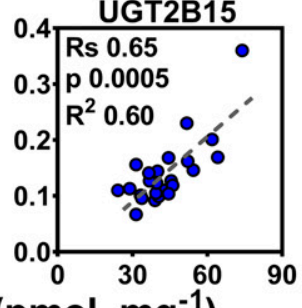

Fig. 3. Correlation between individual UGT enzyme abundances and activity rates ( $n=24)$ using the original dataset (A) and the reassessed data on the basis of the proposed strategy (B). Moderate to strong, statistically significant correlations are shown in blue and weak correlations in gray. Units of abundance-measurement are picomoles per milligram of HLM protein, and units of catalytic activity are

strategy of monitoring the "highest reporter" will probably work well when soluble proteins are quantified; however, for membrane-bound proteins, especially those with unknown structures (e.g., UGTs), a more secure strategy is to monitor "best performer" peptides. Discerning best performers will almost always involve visual examination of MS and MS/MS spectra. For example, isobaric sequences representing UGT1A6 (WIYGVSK) and UGT2B7 (ADVWLIR) overlapped on the chromatogram, leading to overestimation of abundance, especially for UGT1A6. Discrepancies in abundance in relation to peptide choice have previously been reported to reach up to 30-fold in the case of P-glycoprotein in hepatocytes (Chen et al., 2017), and therefore applying suitable criteria is essential to ensure quality of surrogate peptides (Bhatt and Prasad, 2018). Such criteria were previously used in a label-free experiment on matched liver samples, which resulted in good agreement with QconCAT measurements of several UGT enzymes (Achour et al., 2017a).

Because targeted quantification uses MS/MS data as a basis, the properties of fragments are as important as those of parent peptides. Carr et al. (2014) recommended monitoring three to five fragments, allowing inconsistencies in measurements to be reconciled. Fragments are typically selected in silico on the basis of predicted peak intensities rather than sequence properties (Carr et al., 2014). There are several potential pitfalls with this approach. First, MS/MS spectra may have several low intensity peaks (especially with proline-containing peptides), so that a lower number of consistent measurements can be made. Two peptides, YLSIPTVFFLR (UGT1A3) and ESSFDAVFLDPFDNCGLIVAK (UGT1A9), were subject to this error, when peaks of lower quality returned inconsistent ratios. Although optimizing transition selection resulted in improved correlation with activity, quantitative methods for these two enzymes still require improvement. Additionally, erroneous quantification can occur when uniqueness of peptide-to-fragment $m / z$ values cannot be ensured, most often in complex biologic mixtures, with short sequences being most affected (Carr et al., 2014). The MRM filters (retention time, parent ion $\mathrm{m} / \mathrm{z}$, and fragment ion $\mathrm{m} / \mathrm{z}$ ) are normally sufficient to ensure exclusive selection of fragments, but not always, especially in nonscheduled experiments, where retention times are not specified. Peptide sequences WIYGVSK (UGT1A6), ADVWLIR (UGT2B7), and WIYGVSK (UGT2B15) were eventually excluded from analysis for isobaric interference despite returning levels higher than their alternatives. The combined effect of peptide and fragment selection was shown to be substantial (Supplemental Table 1), with abundance values changing 0.5to 3-fold upon reassessment. This led to improved abundance-activity correlation for most UGT enzymes. The initially weak correlation for UGTs 1A4, 1A6, and 2B15 $\left(R \mathrm{~s}=0.19-0.35, P>0.05 ; R^{2}=0.01-0.14\right)$ became moderate $\left(R \mathrm{~s}=0.52-0.56, P<0.01 ; R^{2}=0.24-0.31\right)$. For other UGTs (1A1, 1A3, 2B7), this improved mainly in terms of data scatter (from $R^{2}=0.30$ to $R^{2}=0.47-0.57$ ), although correlation for UGT1A9 remained weak $\left(R \mathrm{~s}=0.20, P=0.33, R^{2}=0.07\right)$.

Effects of Correction for the Quality of Isotopically Labeled Standards. QconCATs are artificial proteins expressed in-house, and the extent of labeling varies, depending on the construct and culture conditions, from $\sim 95-99 \%$ (Achour et al., 2015). This means that QconCATs can contribute unlabeled peptide that may affect quantification when the analyte is expressed at low levels (Carr et al., 2014). Table 1 shows the extent of label incorporation into each peptide, reflecting more efficient labeling of arginines than lysines (Russell et al.,

nanomoles (glucuronide) per minute per milligram of HLM protein. Substrates used for activity measurement are: $\beta$-estradiol (UGT1A1), chenodeoxycholic acid (UGT1A3), trifluoperazine (UGT1A4), 5-hydroxytryptophol (UGT1A6), propofol (UGT1A9), zidovudine (UGT2B7), S-oxazepam (UGT2B15). Rs, Spearman correlation coefficient. Dashed lines represent lines of regression. 


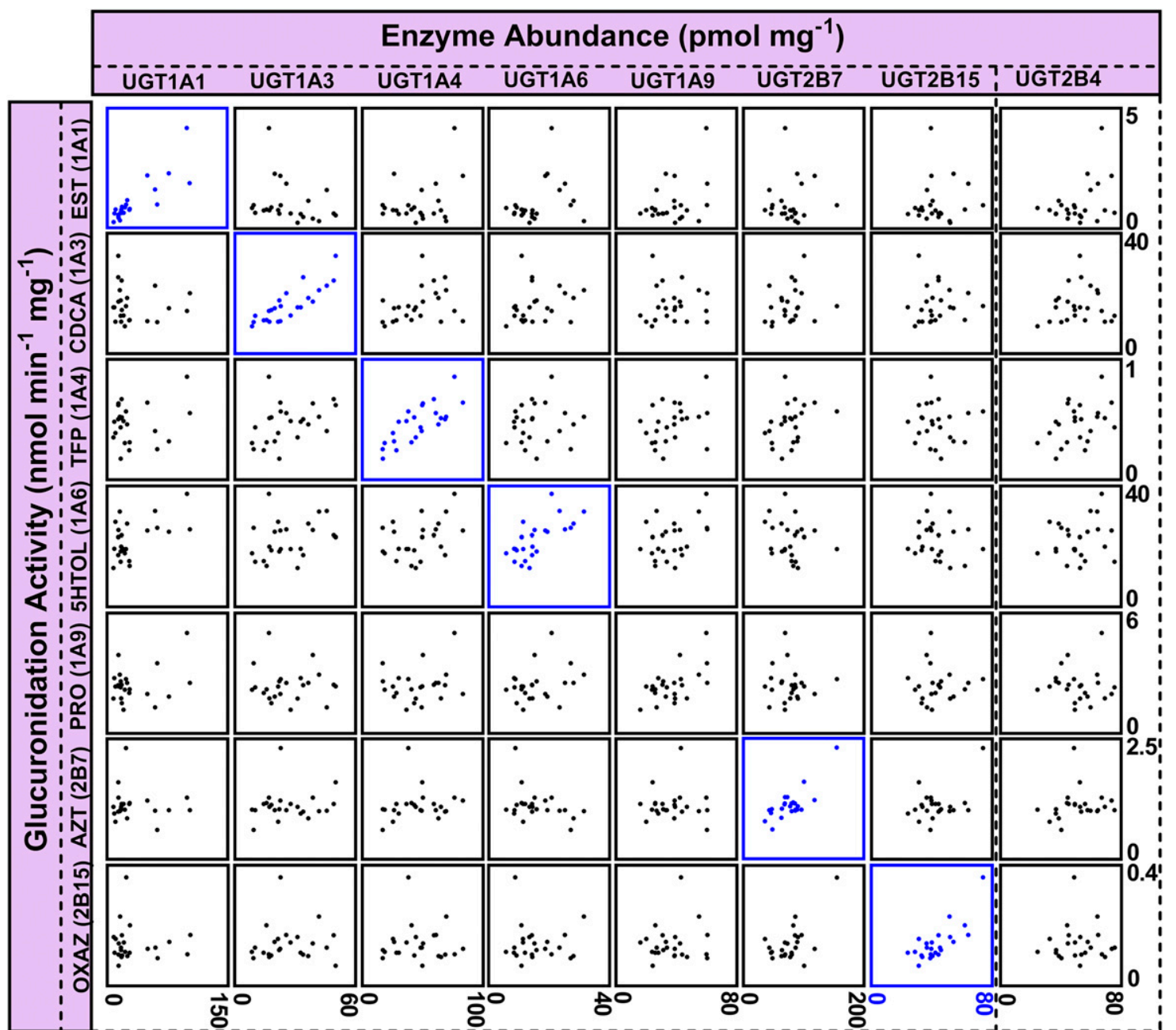

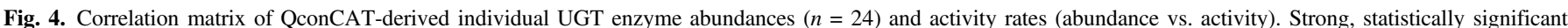

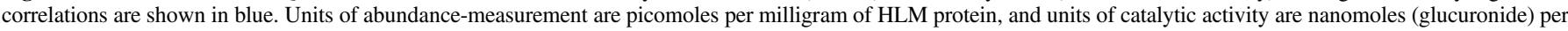

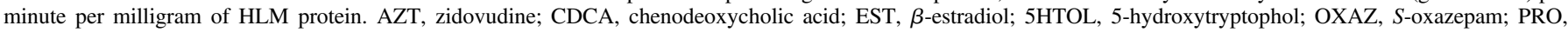
propofol; TFP, trifluoperazine. Supplemental Table 2 shows the statistical analysis used to generate the abundance-activity correlation matrix.

2013). The outcome of correction for purity was that levels of UGTs had a variable artifactual component of up to $10 \%$, with little effect on correlation with activity. A similar trend was observed with efflux transporters BCRP and MRP2, quantified in human jejunum using QconCAT methodology (Harwood et al., 2015), which were overestimated on average by $10 \%$ and $7 \%$, respectively, when corrections for purity were not considered (Harwood et al., 2016a). However, these errors do not always lead to meaningful differences in pharmacokinetic outcomes (Harwood et al., 2016b).

Effects of Normalization of Abundances. Normalization relies on protein content determination using colorimetric assays, which are prone to interference from reagents commonly used in routine sample processing, including detergents, chaotropes, and reducing agents (Wiśniewski and Gaugaz, 2015). In particular, BCA assay is incompatible with commonly used concentrations of urea and dithiothreitol (Smith et al., 1985), whereas Bradford reagent tends to interact with sodium dodecyl sulfate (Bradford, 1976), a detergent used for gel-based sample preparation. A limitation of the cross-laboratory study (Achour et al., 2017b) was the use of Bradford assay with QconCAT measurements, whereas both activity and SIL-based measurements were normalized using BCA assay. Comparing the two protein measurements (Fig. 2C) indicates that, although average protein content in the samples was similar, there was no correlation between individual values. Normalization against the same protein content resulted in changes in enzyme levels reaching up to $50 \%$, with improved correlation with activity, mainly in terms of scatter for six UGTs (from $R^{2}=0.24-0.57$ to $R^{2}=0.48-0.73$ ), and UGT1A9 showed substantial improvement to moderate correlation ( $\left.R \mathrm{~s}=0.47, P=0.02 ; R^{2}=0.34\right)$. It is not clear, however, whether BCAnormalization leads to better results; it may just lead to more consistent error. We have recently illustrated that normalization relative to tissue mass instead of fractional protein introduces less artifactual variability to end-point measurements (Achour et al., 2017a).

Overall Effects of Systematic Reassessment on UGT Measurements. Specific effects of the assessed factors on abundance of each enzyme are described in Supplemental Results. Collectively, the proposed strategy led to 0.5 - to 3.3-fold change in UGT levels, with substantial improvement in correlation with activity (Fig. 3) and tighter levels of interindividual variability in abundance (26\%-86\%), matching variability in activity (27\%-67\%), in line with recent literature (Margaillan et al., 2015). In addition, cross-laboratory comparison of UGT abundances seemed to indicate overall agreement, returning generally interchangeable abundance values (Supplemental Figs. 3 and 4). On the basis of these considerations, a list of established UGTspecific methods was generated (Supplemental Table 4). 
The correlation matrix (Fig. 4) confirms specificity of protein and activity data, with no evidence of spurious abundance-activity relationships. A similar complementary approach was used previously to discern tissue-specific glycolytic and gluconeogenic pathways (Wiśniewski et al., 2015). Expression intercorrelations uncovered in the UGT dataset (Supplemental Table 3) were also in line with literature (Achour et al., 2014a; Margaillan et al., 2015). Correlations of enzyme expression have recently been adopted for more realistic model-based predictions of drug clearance and drug-drug interactions (Barter et al., 2010; Doki et al., 2018), with additional established correlations making their way into commonly used platforms, such as Simcyp.

Conclusions. The QconCAT approach offers several advantages (Al Feteisi et al., 2015a), and therefore it has recently been adopted for various clinical and biologic applications (Dzieciatkowska et al., 2015; Wang et al., 2015; Kito et al., 2016). Although initial applications were primarily aimed to quantify soluble proteins, often in simple organisms, it is clear that QconCAT can be applied to membrane-bound mammalian proteins. However, monitored peptides and fragments need to be chosen carefully, preferably using a priori selection, and corrections are required for relatively low purity standard peptides targeted at low abundance proteins. We continue to advocate using tissue mass for abundance normalization and activity data for quality control. We propose optimized QconCAT methods for the quantification of several UGTs (1A1, 1A6, 2B4, 2B7, and 2B15) and a robust data analysis strategy for targeted proteomic quantification, particularly applicable for QconCAT-based measurements.

\section{Acknowledgments}

The authors thank Dr. Larry Tremaine of Pfizer (Groton, CT) for facilitating the collaboration between the participating laboratories, Dr. John Fallon and Dr. Philip Smith of the University of North Carolina at Chapel Hill for constructive discussions, and Eleanor Savill for assistance with preparation of the manuscript.

\section{Authorship Contributions}

Participated in research design: Achour, Dantonio, Niosi, Novak, Al-Majdoub, Goosen, Rostami-Hodjegan, Barber.

Performed data analysis: Achour, Dantonio, Niosi, Novak, Al-Majdoub.

Wrote or contributed to the writing of the manuscript: Achour, Dantonio, Niosi, Novak, Al-Majdoub, Goosen, Rostami-Hodjegan, Barber.

\section{References}

Achour B, Al Feteisi H, Lanucara F, Rostami-Hodjegan A, and Barber J (2017a) Global proteomic analysis of human liver microsomes: rapid characterization and quantification of hepatic drugmetabolizing enzymes. Drug Metab Dispos 45:666-675.

Achour B, Al-Majdoub ZM, Al Feteisi H, Elmorsi Y, Rostami-Hodjegan A, and Barber J (2015) Ten years of QconCATs: application of multiplexed quantification to small medically relevant proteomes. Int J Mass Spectrom 391:93-104.

Achour B, Dantonio A, Niosi M, Novak JJ, Fallon JK, Barber J, Smith PC, Rostami-Hodjegan A, and Goosen TC (2017b) Quantitative characterization of major hepatic UDP-glucuronosyltransferase enzymes in human liver microsomes: comparison of two proteomic methods and correlation with catalytic activity. Drug Metab Dispos 45:1102-1112

Achour B, Rostami-Hodjegan A, and Barber J (2014a) Protein expression of various hepatic uridine 5 '-diphosphate glucuronosyltransferase (UGT) enzymes and their inter-correlations: a metaanalysis. Biopharm Drug Dispos 35:353-361.

Achour B, Russell MR, Barber J, and Rostami-Hodjegan A (2014b) Simultaneous quantification of the abundance of several cytochrome $\mathrm{P} 450$ and uridine $5^{\prime}$-diphospho-glucuronosyltransferase enzymes in human liver microsomes using multiplexed targeted proteomics. Drug Metab Dispos 42:500-510.

Al Feteisi H, Achour B, Barber J, and Rostami-Hodjegan A (2015a) Choice of LC-MS methods for the absolute quantification of drug-metabolizing enzymes and transporters in human tissue: a comparative cost analysis. AAPS $J$ 17:438-446.

Al Feteisi H, Achour B, Rostami-Hodjegan A, and Barber J (2015b) Translational value of liquid chromatography coupled with tandem mass spectrometry-based quantitative proteomics for in vitro-in vivo extrapolation of drug metabolism and transport and considerations in selecting appropriate techniques. Expert Opin Drug Metab Toxicol 11:1357-1369.

Al-Majdoub ZM, Carroll KM, Gaskell SJ, and Barber J (2014) Quantification of the proteins of the bacterial ribosome using QconCAT technology. J Proteome Res 13:1211-1222.

Barter ZE, Perrett HF, Yeo KR, Allorge D, Lennard MS, and Rostami-Hodjegan A (2010) Determination of a quantitative relationship between hepatic CYP3A5*1/*3 and CYP3A4 expression for use in the prediction of metabolic clearance in virtual populations. Biopharm Drug Dispos 31:516-532.
Bhatt DK and Prasad B (2018) Critical issues and optimized practices in quantification of protein abundance level to determine interindividual variability in DMET proteins by LC-MS/MS proteomics. Clin Pharmacol Ther 103:619-630.

Bradford MM (1976) A rapid and sensitive method for the quantitation of microgram quantities of protein utilizing the principle of protein-dye binding. Anal Biochem 72:248-254.

Brownridge P, Holman SW, Gaskell SJ, Grant CM, Harman VM, Hubbard SJ, Lanthaler K, Lawless C, O'Cualain R, Sims P, et al. (2011) Global absolute quantification of a proteome: challenges in the deployment of a QconCAT strategy. Proteomics 11:2957-2970.

Carr SA, Abbatiello SE, Ackermann BL, Borchers C, Domon B, Deutsch EW, Grant RP, Hoofnagle AN, Hüttenhain R, Koomen JM, et al. (2014) Targeted peptide measurements in biology and medicine: best practices for mass spectrometry-based assay development using a fit-forpurpose approach. Mol Cell Proteomics 13:907-917.

Carroll KM, Simpson DM, Eyers CE, Knight CG, Brownridge P, Dunn WB, Winder CL, Lanthaler K, Pir P, Malys N, et al. (2011) Absolute quantification of the glycolytic pathway in yeast: deployment of a complete QconCAT approach. Mol Cell Proteomics 10:M111. 007633.

Chen B, Liu L, Ho H, Chen Y, Yang Z, Liang X, Payandeh J, Dean B, Hop CECA, and Deng Y (2017) Strategies of drug transporter quantitation by LC-MS: importance of peptide selection and digestion efficiency. AAPS J 19:1469-1478.

Doki K, Darwich AS, Achour B, Tornio A, Backman JT, and Rostami-Hodjegan A (2018) Implications of intercorrelation between hepatic CYP3A4-CYP2C8 enzymes for the evaluation of drug-drug interactions: a case study with repaglinide. Br J Clin Pharmacol DOI: 10.1111/ bcp.13533 [published ahead of print].

Dzieciatkowska M, D’Alessandro A, Hill RC, and Hansen KC (2015) Plasma QconCATs reveal a gender-specific proteomic signature in apheresis platelet plasma supernatants. J Proteomics 120:1-6.

Eyers CE, Lawless C, Wedge DC, Lau KW, Gaskell SJ, and Hubbard SJ (2011) CONSeQuence: prediction of reference peptides for absolute quantitative proteomics using consensus machine learning approaches. Mol Cell Proteomics 10:M110.003384

Fallon JK, Neubert H, Hyland R, Goosen TC, and Smith PC (2013) Targeted quantitative proteomics for the analysis of 14 UGT1As and -2Bs in human liver using NanoUPLC-MS/MS with selected reaction monitoring. J Proteome Res 12:4402-4413.

Guillemette C, Lévesque É, and Rouleau M (2014) Pharmacogenomics of human uridine diphospho-glucuronosyltransferases and clinical implications. Clin Pharmacol Ther 96:324-339.

Harwood MD, Achour B, Neuhoff S, Russell MR, Carlson G, Warhurst G, and Rostami-Hodjegan A (2016a) In vitro-in vivo extrapolation scaling factors for intestinal P-glycoprotein and breast cancer resistance protein: part I: a cross-laboratory comparison of transporter-protein abundances and relative expression factors in human intestine and Caco-2 cells. Drug Metab Dispos 44:297-307.

Harwood MD, Achour B, Neuhoff S, Russell MR, Carlson G, Warhurst G, and Rostami-Hodjegan A (2016b) In vitro-in vivo extrapolation scaling factors for intestinal P-glycoprotein and breast cancer resistance protein: part II. The impact of cross-laboratory variations of intestinal transporter relative expression factors on predicted drug disposition. Drug Metab Dispos 44:476-480.

Harwood MD, Achour B, Russell MR, Carlson GL, Warhurst G, and Rostami-Hodjegan A (2015) Application of an LC-MS/MS method for the simultaneous quantification of human intestinal transporter proteins absolute abundance using a QconCAT technique. J Pharm Biomed Anal 110:27-33.

Kamiie J, Ohtsuki S, Iwase R, Ohmine K, Katsukura Y, Yanai K, Sekine Y, Uchida Y, Ito S, and Terasaki T (2008) Quantitative atlas of membrane transporter proteins: development and application of a highly sensitive simultaneous LC/MS/MS method combined with novel in-silico peptide selection criteria. Pharm Res 25:1469-1483.

Kito K, Okada M, Ishibashi Y, Okada S, and Ito T (2016) A strategy for absolute proteome quantification with mass spectrometry by hierarchical use of peptide-concatenated standards. Proteomics 16:1457-1473.

Lawless C, Holman SW, Brownridge P, Lanthaler K, Harman VM, Watkins R, Hammond DE, Miller RL, Sims PF, Grant CM, et al. (2016) Direct and absolute quantification of over 1800 yeast proteins via selected reaction monitoring. Mol Cell Proteomics 15:1309-1322.

Lawless C and Hubbard SJ (2012) Prediction of missed proteolytic cleavages for the selection of surrogate peptides for quantitative proteomics. OMICS 16:449-456.

Margaillan G, Rouleau M, Klein K, Fallon JK, Caron P, Villeneuve L, Smith PC, Zanger UM, and Guillemette C (2015) Multiplexed targeted quantitative proteomics predicts hepatic glucuronidation potential. Drug Metab Dispos 43:1331-1335.

Pratt JM, Simpson DM, Doherty MK, Rivers J, Gaskell SJ, and Beynon RJ (2006) Multiplexed absolute quantification for proteomics using concatenated signature peptides encoded by QconCAT genes. Nat Protoc 1:1029-1043.

Russell MR, Achour B, Mckenzie EA, Lopez R, Harwood MD, Rostami-Hodjegan A, and Barber J (2013) Alternative fusion protein strategies to express recalcitrant QconCAT proteins for quantitative proteomics of human drug metabolizing enzymes and transporters. J Proteome Res 12:5934-5942.

Scott KB, Turko IV, and Phinney KW (2016) QconCAT: internal standard for protein quantification. Methods Enzymol 566:289-303.

Smith PK, Krohn RI, Hermanson GT, Mallia AK, Gartner FH, Provenzano MD, Fujimoto EK, Goeke NM, Olson BJ, and Klenk DC (1985) Measurement of protein using bicinchoninic acid. Anal Biochem 150:76-85.

Wang H, Zhang H, Li J, Wei J, Zhai R, Peng B, Qiao H, Zhang Y, and Qian X (2015) A new calibration curve calculation method for absolute quantification of drug metabolizing enzymes in human liver microsomes by stable isotope dilution mass spectrometry. Anal Methods 7:5934-5941.

Wegler C, Gaugaz FZ, Andersson TB, Wiśniewski JR, Busch D, Gröer C, Oswald S, Norén A, Weiss F, Hammer HS, et al. (2017) Variability in mass spectrometry-based quantification of clinically relevant drug transporters and drug metabolizing enzymes. Mol Pharm 14:3142-3151. Wiśniewski JR and Gaugaz FZ (2015) Fast and sensitive total protein and peptide assays for proteomic analysis. Anal Chem 87:4110-4116.

Wiśniewski JR, Gizak A, and Rakus D (2015) Integrating proteomics and enzyme kinetics reveals tissue-specific types of the glycolytic and gluconeogenic pathways. J Proteome Res 14: $3263-3273$.

Address correspondence to: Dr. Jill Barber, Centre for Applied Pharmacokinetic Research, Division of Pharmacy and Optometry, School of Health Sciences, University of Manchester, Stopford Building, Oxford Road, Manchester, M13 9PT, United Kingdom. E-mail: Jill.Barber@manchester.ac.uk 